\title{
Soft Robotics: Cerebellar Inspired Control of Artificial Muscles
}

\author{
E. D. Wilson ${ }^{1}$, T. Assaf ${ }^{3}$, M. J. Pearson ${ }^{3}$, J. M. Rossiter ${ }^{3}$ P. Dean ${ }^{1}$, S. R. Anderson ${ }^{1,2}$, J. Porrill ${ }^{1}$ \\ ${ }^{1}$ Sheffield Center for Robotics, University of Sheffield \\ ${ }^{2}$ Department of Automatic Control and Systems Engineering, Faculty of Engineering, University of Sheffield. \\ ${ }^{3}$ Bristol Robotics Laboratory, University of Bristol and University of the West of England.
}

\begin{abstract}
Soft robots have the potential to greatly improve human-robot interaction via intrinsically safe, compliant designs. However, new compliant materials used in soft robotics - artificial muscles - are fabricated with poor tolerances and have time-varying dynamics. Therefore, a key technical challenge is to develop adaptive control algorithms for these materials. Here, we take a novel bioinspired approach to artificial muscle control using the adaptive filter model of the cerebellum. The cerebellum is a brain structure essential for fine-tuning human performance in a diverse range of sensory and motor tasks. Its ability to automatically calibrate and adapt to changes in a wide variety of systems using a homogenous, repeating structure suggests that cerebellar-inspired models are highly suited to controlling artificial muscles in a range of tasks. We investigate the performance of the cerebellar adaptive filter algorithm in the displacement control of a soft actuator. Experimental results demonstrate that the cerebellar algorithm is successful and learns to accurately control the time-varying dynamics of the soft actuator in real-time.
\end{abstract}

Keywords Artificial Muscle; Adaptive Control; Cerebellum

\section{INTRODUCTION}

The new field of soft robotics offers the prospect of replacing traditional rigid robots with soft compliant counterparts more suited to unstructured environments and human centred robotics [1]. Recently, artificial muscles such as electro-active polymers (EAPs) have been developed in an attempt to realise new soft-actuated robotic systems. These artificial muscles seek to emulate the scalability, high mechanical compliance, versatility and large strain capability of biological muscle. A particular class of EAP, dielectric EAP (DEAP) can be used to manufacture compliant actuators with high energy density, large strain capability, and a relatively fast response [2]. The DEAP class of actuator has great potential for soft robotics. However, DEAPs present a number of control challenges; they have non-linear dynamics, are manufactured with large tolerances, and are subject to creep and time related aging. Suitable control schemes for these actuators are currently lacking.

Taking inspiration from neural control would seem to be a natural strategy for artificial muscle (DEAP) control since the control challenges are similar to those posed by biological muscle. The cerebellum is a region of the brain strongly associated with adaptive control and skilled movement. Cerebellar-inspired algorithms have a track record of success in other robotic applications [3]. Here, we use the adaptive filter model of the cerebellum [4] to control a simple onedegree of freedom DEAP actuator.

In summary, we first present the cerebellar adaptive filter algorithm then apply this algorithm to the real time displacement control of a DEAP actuator.

\section{Cerebellar Algorithm}

The cerebellar microcircuit can be modelled as an adaptive filter [4]. A control architecture showing the cerebellar contribution to motor plant compensation (based on the Vestibular Ocular Reflex) is given in Fig. 1.

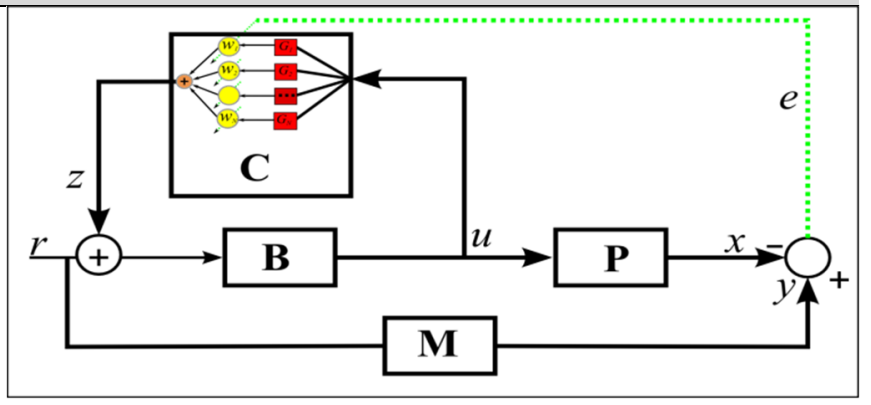

Figure 1. Cerebellar based control scheme. $\mathrm{P}$ is the plant being controlled, $B$ a fixed feed-forward brainstem controller, $M$ a reference model and $C$ the adaptive cerebellar filter.

This cerebellar inspired control scheme was applied to the displacement control of a DEAP. Adaptive filter weights were learnt using the covariance learning rule (equivalent to least mean squared learning). This approximately minimises the sensory error (i.e. Difference in actual and desired displacement for the DEAP).

\section{EXPERIMENTAL DETAILS}

Our DEAP actuators are comprised of a thin passive elastomer film (3M VHB 4905) sandwiched between two compliant electrodes (carbon grease, MG chemicals). In response to an applied voltage the electrodes squeeze the film in the thickness direction, resulting in biaxial expansion. In order to constrain the controlled variable to one degree of freedom a spherical load was placed at the centre of the actuator and its motion in the vertical plane measured and controlled. The experimental set up is summarised in Fig. 2. 


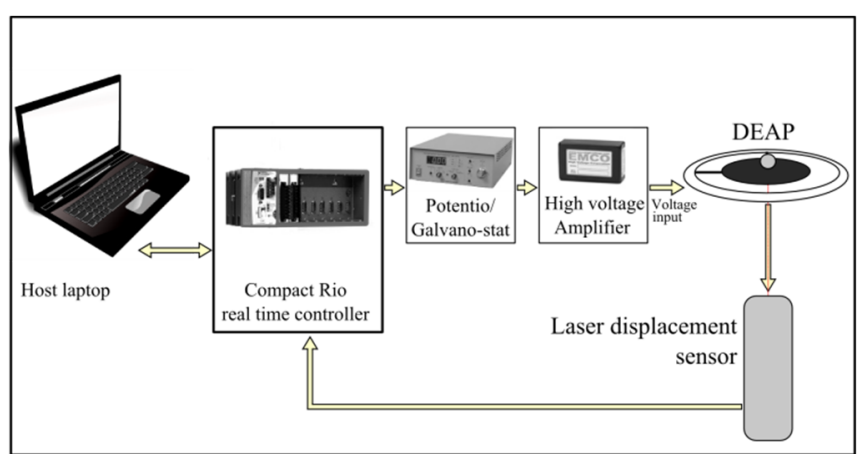

Figure 2. Summary of experimental set up for real time DEAP control. The control algorithm is implemented on a Compact Rio.

\section{RESULTS}

The cerebellar control algorithm was applied to the real time control of a DEAP. The fixed feed-forward controller, B was set to provide compensation for the average behaviour of six different actuators. The reference signal, $r$ was a collared noise signal with frequency range $0-1 \mathrm{~Hz}$ and amplitude range $0.2-0.65$ (the amplitude range was constrained to give a linear response).
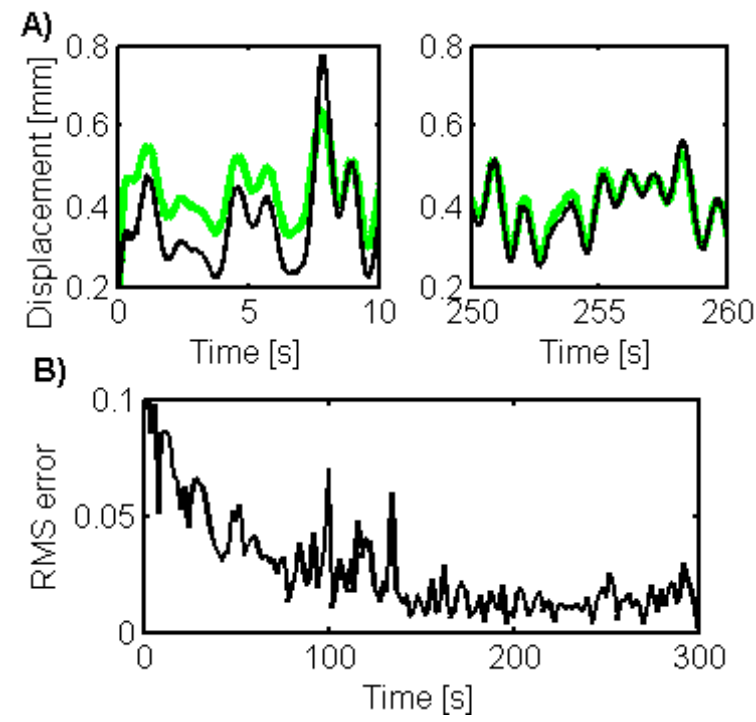

Figure 3. Experimental control of DEAP using adaptive cerebellar controller; (a). Desired ( $\square$ ) and actual $(\square)$ displacement at start and end of learning; (b) Windowed RMS errors during learning.

Fig. 3 shows the displacement control results for one DEAP. The cerebellar adaptive filter is able to compensate for the fabrication differences across DEAPs and any time-varying dynamics.

\section{DISCUSSION}

DEAPs show considerable changes in their dynamics over time and across different actuators. Time varying changes are likely to occur due to creep and time-related aging and differences across actuators due to poor fabrication tolerances. In order to realise the potential of these actuators for soft robotic systems control algorithms capable of compensating for changes in the plant dynamics are required. The cerebellar algorithm is able to accurately track the displacement response of a DEAP in spite of these dynamic plant changes.

The ability of the cerebellum to calibrate and adapt to changes in a wide variety of systems using a relatively homogenous structure suggests that the control algorithm presented here has the potential to provide a modular controller for soft robotics in generic control tasks. For example, it could also be used to control the force, or impedance response, or to calibrate tasks. However to be more generally applicable the algorithm must be extended to the control of non-linear, multi-degree of freedom systems.

\section{CONCLUSIONS}

In this contribution we have demonstrated the suitability of a cerebellar control algorithm for real-time tracking control of a DEAP actuator. This work is the first step in developing a 'cerebellar chip' algorithm capable of controlling a wide range of sensory and motor tasks. To realise this the cerebellar algorithm needs to be extended to the control of non-linear plants and multi-degree of freedom systems.

\section{ACKNOWLEDGEMENTS}

Work was funded by an EPSRC grant no. EP/IO32533/1

\section{REFERENCES}

1. Majidi, C. Soft Robotics: A Perspective-Current Trends and Prospects for the Future. Soft Robotics 1(1): 5-11, 2014.

2. O'Halloran, A., O'Malley, F., McHugh, P. A review on dielectric elastomer actuators, technology, applications, and challenges. J. Appl. Phys, 104(7), 071101, 2008.

3. Lenz, A., Anderson, S.R., Pipe, A.G., Melhuish, C., Dean, P., Porrill, J. Cerebellar-Inspired Adaptive Control of a Robot Eye Actuated by Pneumatic Artificial Muscles. IEEE T. Syst. Man, Cy. B, 39(6): 1420-1433

4. Dean, P., Porrill, J., Ekerot, C.-F., Jörntell, H. The Cerebellar Microcircuit as an Adaptive Filter: Experimental and Computational Evidence Nature Reviews Neuroscience 11, 30$\underline{43,2010}$ 